González Rojas, D., Cifuentes Sánchez, V. J., Sancho Miró, A. (2019): “Las infraestructuras de datos espaciales al servicio de la gestión del agua. El caso de la Confederación Hidrográfica del Guadalquivir”, GeoFocus (Artículos), $n^{\circ}$ 24, p.3-17. ISSN: 1578-5157 http://dx.doi.org/10.21138/GF.548

\title{
LAS INFRAESTRUCTURAS DE DATOS ESPACIALES AL SERVICIO DE LA GESTIÓN DEL AGUA. EL CASO DE LA CONFEDERACIÓN HIDROGRÁFICA DEL GUADALQUIVIR
}

\author{
DAVID GONZÁLEZ ROJAS ${ }^{1}$, VÍCTOR JUAN CIFUENTES SÁNCHEZ², ALFONSO SANCHO \\ MIRÓ $^{3}$ \\ ${ }^{1,2,3}$ Confederación Hidrográfica del Guadalquivir. Oficina de Planificación Hidrológica \\ Plaza de España, Sector II y Sector III, C.P 41071 Sevilla, España \\ 1․ㅣㅇㅡalez@chguadalquivir.es, ${ }^{2}$ vjcifuentes@chguadalquivir.es, \\ ${ }^{3}$ asancho@chguadalquivir.es
}

\section{RESUMEN}

La Confederación Hidrográfica del Guadalquivir tiene entre sus competencias la planificación hidrológica de los recursos hídricos que discurren por las demarcaciones del Guadalquivir, Ceuta y Melilla, la realización de obras de interés general, así como la gestión del Dominio Público Hidráulico. Esto precisa una ingente cantidad de datos relativos al agua y al territorio, que son representados sobre cartografía digital. La Confederación Hidrográfica del Guadalquivir ha trabajado en la construcción de una Infraestructura de Datos Espaciales que, además de aglutinar información cartográfica y alfanumérica de calidad contrastada, ofrece servicios remotos de acceso a los datos geoespaciales. El fin último de la IDE-CHG es facilitar el conocimiento de la compleja realidad geográfica del territorio gestionado y potenciar las relaciones con los usuarios a través de una mayor presencia en la web, siguiendo las recomendaciones de la guía GIS de la Directiva Marco del Agua y respondiendo a los requisitos de la Directiva INSPIRE.

Palabras clave: Confederación Hidrográfica del Guadalquivir, Infraestructura de Datos Espaciales, Geoportal, Directiva Marco del Agua. 
González Rojas, D., Cifuentes Sánchez, V. J., Sancho Miró, A. (2019): “Las infraestructuras de datos espaciales al servicio de la gestión del agua. El caso de la Confederación Hidrográfica del Guadalquivir”, GeoFocus (Artículos), $n^{\circ}$ 24, p.3-17. ISSN: 1578-5157 http://dx.doi.org/10.21138/GF.548

\section{SPATIAL DATA INFRASTRUCTURES FOR WATER MANAGEMENT. THE CASE OF GUADALQUIVIR RIVER BASIN AUTHORITY}

\section{ABSTRACT}

The Guadalquivir River Basin Authority have, among its responsibilities, the hydrological planning of water resources, which flow through the demarcation of the Guadalquivir, Ceuta and Melilla, the execution of works of general interest as well as the management of Hydraulic Public Domain. This requires an enormous amount of data related to water and territory, which are represented on digital cartography. The Guadalquivir River Basin Authority has worked in the construction of a Spatial Data Infrastructure that, in addition to bringing together cartographic and alphanumeric proven quality information, provides remote access to geospatial data. The final goal of the IDE-CHG is to facilitate the understanding of the complex geographic reality of the watershed and enhance relationships with users through a greater presence on the web, following the recommendations of the GIS guide in the Water Framework Directive, and responding to the requirements of the INSPIRE directive.

Keywords: Guadalquivir River Basin Authority, Spatial Data Infrastructure, Geoportal, Water Framework Directive.

\section{Introducción}

La Confederación Hidrográfica del Guadalquivir es el organismo responsable de la gestión del agua en la demarcación hidrográfica del Guadalquivir, así como en las demarcaciones hidrográficas de Ceuta y de Melilla. Esta institución fue creada en 1927 como una entidad de derecho público con personalidad jurídica propia para administrar las aguas del Guadalquivir, cuya cuenca se extienden por las comunidades autónomas de Andalucía, Extremadura, Castilla-La Mancha y Murcia. La Confederación Hidrográfica del Guadalquivir está adscrita a efectos administrativos al Ministerio de Agricultura y Pesca, Alimentación y Medio Ambiente, del que depende como Organismo Autónomo con plena autonomía funcional.

Entre sus competencias se encuentra la planificación hidrológica, requerimiento legal que se establece con los objetivos generales (artículo 40 del Texto Refundido de la Ley de Aguas) de conseguir el buen estado y la adecuada protección de las masas de agua de la demarcación, la satisfacción de las demandas de agua y el equilibrio y armonización del desarrollo regional y sectorial. Estos objetivos han de alcanzarse incrementando las disponibilidades del recurso, protegiendo su calidad, economizando su empleo y racionalizando sus usos en armonía con el medio ambiente y los demás recursos naturales.

Desde la Unión Europea, a través de la Directiva 2000/60/CE de 23 de octubre de 2000 por la que se establece un marco comunitario de actuación en el ámbito de la política de aguas, en adelante DMA, se ha consolidado la idea de que una nueva cultura del agua es necesaria en la gestión de los recursos, con el objetivo de alcanzar el buen estado de las aguas y ecosistemas acuáticos. Esta filosofía de respeto al medioambiente, en consonancia con la satisfacción de las demandas existentes, es la que se ha ido promoviendo desde la Administración hidráulica durante los últimos años. 
González Rojas, D., Cifuentes Sánchez, V. J., Sancho Miró, A. (2019): "Las infraestructuras de datos espaciales al servicio de la gestión del agua. El caso de la Confederación Hidrográfica del Guadalquivir”, GeoFocus (Artículos), $n^{\circ}$ 24, p.3-17. ISSN: 1578-5157 http://dx.doi.org/10.21138/GF.548

Para cumplir con los requerimientos de la DMA, la legislación española ha modificado y ha adaptado los objetivos de la planificación hidrológica, que debe tratar de compatibilizar la consecución del buen estado de las aguas superficiales y subterráneas con atender las demandas, mediante una gestión racional y sostenible. Además, el artículo 87 del Reglamento de Planificación Hidrológica (Real Decreto 907/2007, de 6 de julio) indica que el Comité de Autoridades Competentes de la demarcación promoverá la elaboración y mantenimiento de un sistema de información sobre el estado de las masas de agua que permita obtener una visión general del mismo, teniendo en cuenta también los objetivos ambientales específicos de las zonas protegidas. Este sistema de información, además de constituir un elemento básico para la planificación y elaboración de los programas de medidas, se utilizará para el seguimiento del plan hidrológico.

Estas tareas requieren una ingente cantidad de datos relativos al agua y al territorio, que son representados sobre cartografía digital. La solicitud de esta información por parte de los ciudadanos y las diferentes instituciones requiere una importante movilización de recursos. Es por ello que, durante los últimos años se ha trabajado en la construcción de un sistema que, además de aglutinar la información cartográfica y alfanumérica de calidad contrastada, ofrezca servicios remotos de acceso a los datos geoespaciales conforme a los principales estándares internacionales. Nos referimos fundamentalmente a las especificaciones del Open Geospatial Consortium (OGC) y de la serie de normas 19100 de la Internacional Organization for Standarization (ISO).

La Infraestructura de Datos Espaciales de la Confederación Hidrográfica del Guadalquivir (http://www.chguadalquivir.es/idechg), en adelante IDE-CHG, nació en el marco del Plan de Modernización Tecnológica del organismo. Uno de los objetivos del citado plan era mejorar el conocimiento de la realidad gestionada, las demarcaciones hidrográficas del Guadalquivir, Ceuta y Melilla, mediante la implantación de un Sistema de Información Geográfica corporativo (González Rojas y Sancho Miró, 2010).

Asimismo, de cara a la implementación de las especificaciones de la Directiva 2007/2/CE del 14 de marzo 2007 por la que se establece un Infraestructura Espacial en la Comunidad Europea (INSPIRE - Infrastructure for Spatial Information in Europe) y de su ley de transposición al ámbito nacional (Ley 14/2010, de 5 de julio, sobre las infraestructuras y los servicios de información geográfica en España), se ha prestado atención a las recomendaciones del Grupo de Trabajo de la Infraestructura de Datos Espaciales de España (GT-IDEE).

En resumen, la IDE-CHG pretende facilitar el acceso a la información espacial, tanto a nivel institucional como a nivel de los propios ciudadanos, así como optimizar la toma de decisiones dentro del organismo. El fin último de la IDE-CHG es facilitar el conocimiento de la compleja realidad geográfica de la cuenca y potenciar las relaciones con los usuarios a través de una mayor presencia en la web, haciendo uso de un conjunto de estándares, protocolos y especificaciones de interoperabilidad. La IDE-CHG es nodo de la IDE de España (http://www.idee.es/) y de la IDE de Andalucía (http://www.ideandalucia.es/).

En el presente artículo se pone de manifiesto la utilidad de las infraestructuras de datos espaciales y la información geográfica en particular, en la gestión de los recursos hídricos. Tiene como objetivo la descripción detallada del sistema implantado en la Confederación Hidrográfica del Guadalquivir. En primer lugar se realiza una revisión de los antecedentes y componentes fundamentales de la IDE-CHG, en cuanto a servicios y aplicaciones. Finalmente, se realiza una descripción de la información geográfica ofrecida en la IDE-CHG. 
González Rojas, D., Cifuentes Sánchez, V. J., Sancho Miró, A. (2019): "Las infraestructuras de datos espaciales al servicio de la gestión del agua. El caso de la Confederación Hidrográfica del Guadalquivir”, GeoFocus (Artículos), $n^{\circ}$ 24, p.3-17. ISSN: 1578-5157 http://dx.doi.org/10.21138/GF.548

\section{Antecedentes}

La implementación de un Sistema de Información Territorial Corporativo para la gestión y consulta de la información geoespacial de la cuenca nació en el marco del Plan de Modernización Tecnológica de la Confederación Hidrográfica del Guadalquivir, cuyos objetivos eran facilitar la gestión integral de los recursos con herramientas que proporcionasen una visión global y en detalle del territorio y de la gestión administrativa y económica asociada y desarrollar nuevos canales de relación con el ciudadano, que le permitieran participar de forma activa en los procesos de gestión del agua mediante la implantación de la Administración Electrónica (Zabala et al. 2006).

Como punto de partida, se estableció una plataforma tecnológica centralizando toda la información espacial demandada por el organismo en un repositorio común y bajo un modelo de datos único al cual accedían los usuarios de forma rápida y sencilla evitando la duplicidad de la información. Esta plataforma estaba basada en la adopción de Oracle como base de datos corporativa, dentro de una arquitectura de desarrollo a tres capas bajo la plataforma J2EE. Uno de los aspectos a destacar de esta definición fue la apuesta por el software libre, estableciéndose su preferencia de uso en caso de igualdad de características técnicas con las alternativas propietarias.

La plataforma web denominada Hidrob@se estaba pensada para satisfacer la gran demanda de consultas básicas y visualización de datos espaciales existente en el organismo, así como para ofrecer personalizaciones específicas y sencillas a los usuarios de los diferentes servicios, tales como la elaboración de informes catastrales, la explotación de las series temporales registradas por las redes de control, etc.

\section{Servicios y arquitectura tecnológica del sistema IDE-CHG}

Una vez implementado en la Confederación Hidrográfica del Guadalquivir el conjunto de aplicaciones que permitían gestionar y explotar coherentemente la información geográfica aportada por los diferentes servicios, el siguiente paso lógico fue adoptar soluciones IDE que permitieran publicar externamente esa información a bajo coste y de manera interoperable. Para ello, se diseñó e implementó un geoportal que actúa como el punto de acceso telemático que el organismo pone a disposición de los ciudadanos para que accedan a la información geográfica relativa al territorio gestionado. A través del geoportal, la Confederación Hidrográfica del Guadalquivir establece, gestiona y provee de acceso a una red de servicios que permite:

- Descubrir datos geográficos.

- Localizar datos geográficos.

- Visualizar datos geográficos.

- Descargar datos geográficos.

La IDE-CHG está basada en tres componentes fundamentales: la geodatabase, el servidor de mapas y las aplicaciones web. El entorno tecnológico base consiste en dos servidores Linux CentOS, contando cada uno de ellos con una instalación del servidor de mapas y con un servidor web Apache Tomcat 6.

La base de datos espaciales está implementada mediante un sistema gestor de bases de datos Oracle versión 10, con extensión Oracle Spatial. Los datos se introducen mediante la 
González Rojas, D., Cifuentes Sánchez, V. J., Sancho Miró, A. (2019): "Las infraestructuras de datos espaciales al servicio de la gestión del agua. El caso de la Confederación Hidrográfica del Guadalquivir”, GeoFocus (Artículos), $n^{\circ}$ 24, p.3-17. ISSN: 1578-5157 http://dx.doi.org/10.21138/GF.548

herramienta ArcSDE de ESRI. En la geodatabase se encuentran todas las capas existentes en la IDE-CHG, tanto las que están publicadas en el visor como las que no. La geodatabase es accesible únicamente desde las oficinas de la Confederación Hidrográfica del Guadalquivir, bien mediante ArcSDE con ArcGIS o gvSIG o bien directamente con gvSIG o QGIS.

El servidor de mapas se ha implementado mediante la aplicación Mapserver, que se encarga de leer la información espacial de la base de datos y servirla en diferentes formatos. Mapserver es una plataforma de código abierto para la publicación de datos espaciales y aplicaciones cartográficas interactivas para la web (Kropla, 2005). El Comité Directivo del Proyecto de MapServer, avalado por Open Source Geospatial Foundation (OSGeo), gestiona y administra el proyecto, el cual es mantenido, mejorado y apoyado por desarrolladores de todo el mundo.

Mapserver es multiplataforma, proporciona una salida cartográfica avanzada, implementa los estándares OGC y permite la compatibilidad con INSPIRE en sus versiones más recientes. Además soporta múltiples formatos y permite proyección de mapa al vuelo mediante la librería Proj4. La elección de Mapserver para la implementación de los servicio WMS, WFS y WCS se produjo tras estudiar detenidamente las dos alternativas que se plantearon: Mapserver y Geoserver. Tras realizar pruebas de rendimiento de ambos servicios, se comprobó que Mapserver ofrecía mejores resultados en cuanto al tiempo de respuesta.

En la Confederación Hidrográfica del Guadalquivir se ha compilado e instalado en un entorno Linux la versión Mapserver 6.4.1 con compatibilidad con Oracle Spatial. Previamente fue necesaria la instalación de una serie de librerías en el sistema operativo como libpng, freetype, libjpeg, zlib, libproj, libcurl, OGR y GDAL. Una vez compilado e instalado, se crearon los ficheros necesarios para implementar los servicios. En nuestro caso, hemos utilizado Mapserver para implementar WMS (Web Map Service) versión 1.3.0, WFS (Web Feature Service) versión 1.0 y WCS (Web Coverage Service) versión 1.0.

Posteriormente se compiló e instaló Mapcache, versión 1.2.1. Mapcache es un servidor que implementa el cacheado de capas WMS mediante rejillas de tamaño configurable. Hace de intermediario entre el peticionario y Mapserver, cacheando las peticiones según la configuración realizada y, posteriomente, sirviendo las capas que tenga cacheadas. Es un servicio totalmente transparente al usuario, ya que a ojos de éste la interacción es idéntica a la que se produciría con Mapserver. Mapcache se configura mediante un fichero en formato XML donde establecemsos qué capas queremos cachear, en qué formato, escala, etc. La utilización de Mapcache mejora enormemente la velocidad de respuesta al usuario.

La IDE-CHG cuenta con tres aplicaciones web: visualizador cartográfico, catálogo de metadatos y buscador de topónimos. Además, se dispone de una zona de contacto, en la que el ciudadano puede realizar las preguntas, sugerencias o consultas que estime oportunas.

Por otro lado, se ha desarrollado una zona de descarga, accesible desde el geoportal, a través de la cual los ciudadanos pueden elegir la capa que les interese, consultar los productos de descarga disponibles para ese dato y descargarlos. Dichos productos hacen referencia a ficheros de ArcGIS (shapefiles), metadatos (adaptados a la plantilla del Núcleo Español de Metadatos), mapas temáticos (diseñados en PDF y JPG) y ficheros de Google Earth (KMZ). 
González Rojas, D., Cifuentes Sánchez, V. J., Sancho Miró, A. (2019): “Las infraestructuras de datos espaciales al servicio de la gestión del agua. El caso de la Confederación Hidrográfica del Guadalquivir”, GeoFocus (Artículos), $n^{\circ}$ 24, p.3-17. ISSN: 1578-5157 http://dx.doi.org/10.21138/GF.548

\subsection{Visualizador cartográfico}

El cliente de visualización de la IDE-CHG (ver figura 1) permite la consulta y personalización de diversas capas de información almacenadas en la base de datos del organismo, ofreciendo a los usuarios una visión integradora del territorio gestionado. Además, brinda la posibilidad de consultar datos geográficos ubicados en servidores externos, que se encuentran en diversos formatos y poseen diferentes sistemas de coordenadas, siempre que éstos cumplan las especificaciones del OGC relativas a Servicios Web de Mapas (WMS).
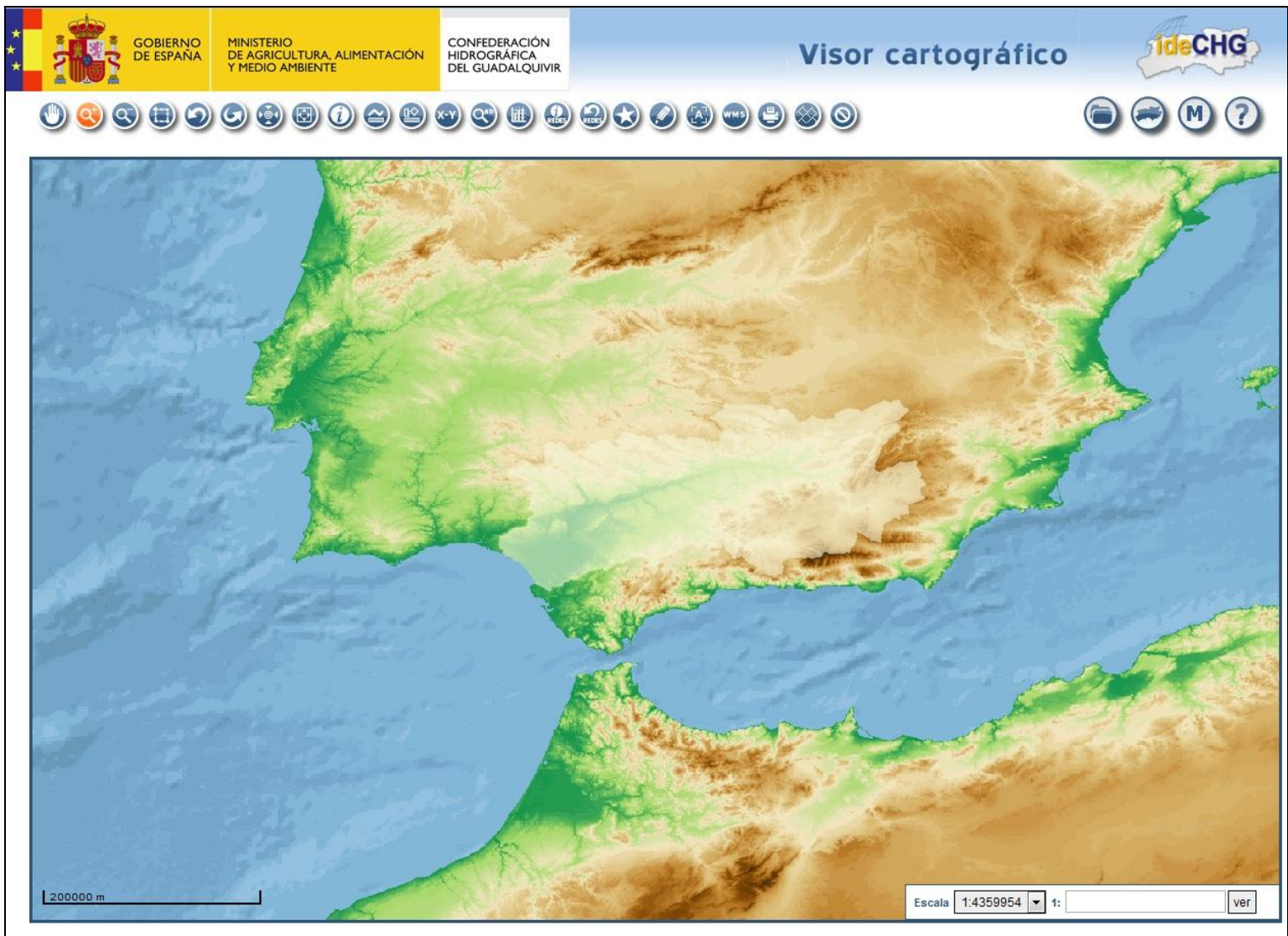

Figura 1. Visor cartográfico de la IDE-CHG.

Fuente: elaboración propia.

Este cliente es una herramienta pensada para usuarios que necesiten procesamiento básico o medio de información geoespacial. Permite superponer capas, ubicar puntos, consultar información sobre las capas, realizar mediciones así como consultar datos de las redes de control, búsquedas alfanuméricas, añadir capas externas, entre otras funcionalidades. Además del visor general, existen tres específicos para las redes de control y para el registro de zonas protegidas.

El visualizador cartográfico es una aplicación desarrollada en la plataforma JavaEE, siguiendo el patrón arquitectónico modelo-vista-controlador, implementando para ello el framework Spring. Por su parte, gran parte de la capa de presentación está delegada en el cliente, mediante la 
González Rojas, D., Cifuentes Sánchez, V. J., Sancho Miró, A. (2019): "Las infraestructuras de datos espaciales al servicio de la gestión del agua. El caso de la Confederación Hidrográfica del Guadalquivir”, GeoFocus (Artículos), $n^{\circ}$ 24, p.3-17. ISSN: 1578-5157 http://dx.doi.org/10.21138/GF.548

tecnología OpenLayers. OpenLayers es una biblioteca de JavaScript de código abierto para mostrar mapas interactivos en los navegadores web y ofrece una interfaz para acceder a diferentes fuentes de información cartográfica en la red. Además, se beneficia del sistema de cacheo de capas, de forma que si una capa está cacheada, no será necesario volver a consultarla.

En términos generales, el visor cartográfico permite ejecutar las siguientes operaciones:

- Realizar funciones básicas de visualización (acercar, alejar, desplazar, etc.).

- Consultar la información de las capas y de las tablas alfanuméricas relacionadas.

- Medir distancias y superficies sobre el mapa.

- Modificar el orden de visualización de los datos.

- Personalizar las propiedades visuales de las capas (color, grosor, transparencia, etiquetado, etc.).

- Imprimir la zona visualizada.

- Realizar consultas alfanuméricas empleando operadores booleanos.

- Realizar búsquedas por coordenadas.

- Consultar las series históricas de las redes de control del organismo.

\subsection{Catálogo de metadatos}

El cliente de búsqueda de metadatos (ver figura 2) permite la exploración de la información residente en la base de datos del organismo, mediante búsquedas directas (por cadenas de caracteres) o búsquedas por categorías (exploración en un árbol temático).

La búsqueda directa proporciona al usuario la opción de realizar búsquedas empleando filtros alfanuméricos (palabras clave, categorías y escalas). Por su parte, la búsqueda por categorías ordena los metadatos de la información en función de las recomendaciones de la directiva INSPIRE, separándolos en conjuntos de datos, series y servicios. En ambos casos, se ofrecen varias opciones al usuario. Por un lado, es posible consultar los metadatos de la información (NEM - ISO 19115) y, por otro, descargar los ficheros shapefile disponibles asociados a la información de interés.

El buscador de metadatos está dividido en dos secciones: búsquedas directas y búsquedas por categorías. La primera proporciona al usuario la opción de realizar búsquedas empleando filtros alfanuméricos (palabras clave, categorías y escala) y/o temporales, en función de la fecha de creación, edición, publicación, etc. de los datos. Por su parte, la búsqueda por categorías ordena los metadatos de la información en grupos temáticos, permitiendo ver la información contenida en dichos grupos. 
González Rojas, D., Cifuentes Sánchez, V. J., Sancho Miró, A. (2019): "Las infraestructuras de datos espaciales al servicio de la gestión del agua. El caso de la Confederación Hidrográfica del Guadalquivir”, GeoFocus (Artículos), $n^{\circ}$ 24, p.3-17. ISSN: 1578-5157 http://dx.doi.org/10.21138/GF.548

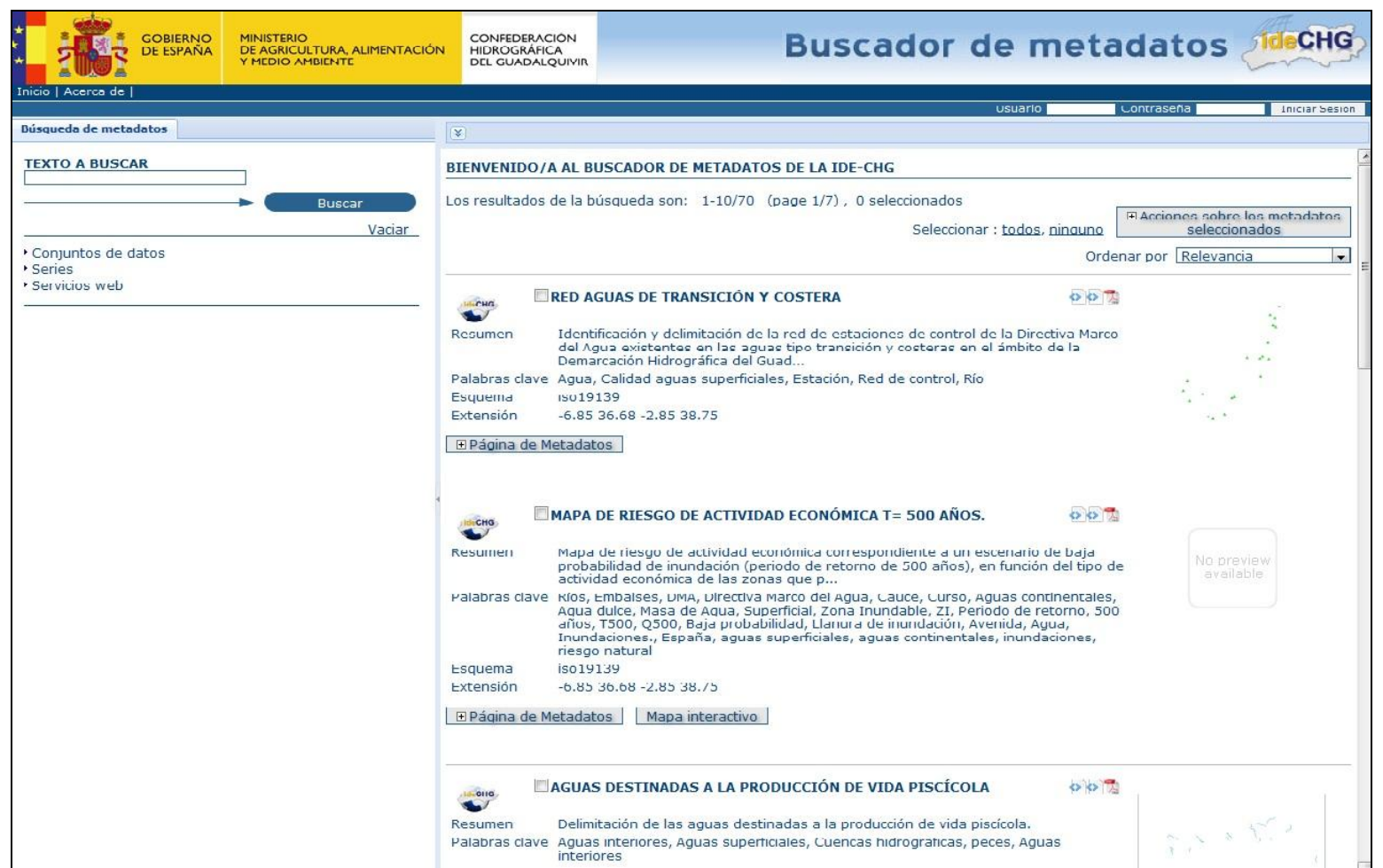

Figura 2. Catálogo de metadados de la IDE-CHG.

Fuente: elaboración propia.

Para la catalogación se ha utilizado la herramienta CatMDEdit. En algunos casos, los metadatos se han creado partiendo de cero y en otros casos, que ya existían metadatos aunque en otro formato, se han transformado, también mediante el uso de CatMDEdit a ISO 19115.

El catálogo de metadatos se ha implementado mediante el software Geonetwork, versión 2.6.4., aplicación informática de software libre y código abierto de catalogación para recursos referenciados al espacio geográfico. Se trata de un entorno de gestión de información espacial estandarizado y descentralizado, diseñado para permitir acceso a bases de datos georreferenciadas, productos cartográficos y metadatos de varias fuentes, mejorando el intercambio y el compartir entre organizaciones y su audiencia, usando las capacidades de la web.

Geonetwork permite incorporar ficheros XML así como imágenes en miniatura de los metadatos. Se han introducido los metadatos de todas las capas así como de los servicios. Geonework es la aplicación encargada de ofrecer el servicio CSW (Catalog Web Service), versión 2.0, que define una interfaz común para la recuperación, captura y consulta de metadatos referentes a datos, servicios y recursos geográficos. 
González Rojas, D., Cifuentes Sánchez, V. J., Sancho Miró, A. (2019): “Las infraestructuras de datos espaciales al servicio de la gestión del agua. El caso de la Confederación Hidrográfica del Guadalquivir”, GeoFocus (Artículos), $n^{\circ}$ 24, p.3-17. ISSN: 1578-5157 http://dx.doi.org/10.21138/GF.548

\subsection{Buscador de topónimos}

El cliente de búsqueda de topónimos ofrece la posibilidad de localizar espacialmente entidades hidrológicas a través de una consulta parametrizable sobre los topónimos. Para tal efecto, el usuario puede realizar búsquedas de:

- Topónimos que se denominen exactamente de una manera o contengan parte de un nombre.

- Topónimos que se encuentren en un municipio concreto.

- Topónimos pertenecientes a una tipología.

- Topónimos localizados dentro de unas coordenadas específicas, etc.

La ventana principal de la herramienta está dividida en dos zonas principales. La parte izquierda, donde se presentan los diferentes criterios de búsqueda y la derecha, reservada para mostrar el resultado de la búsqueda (ver figura 3).

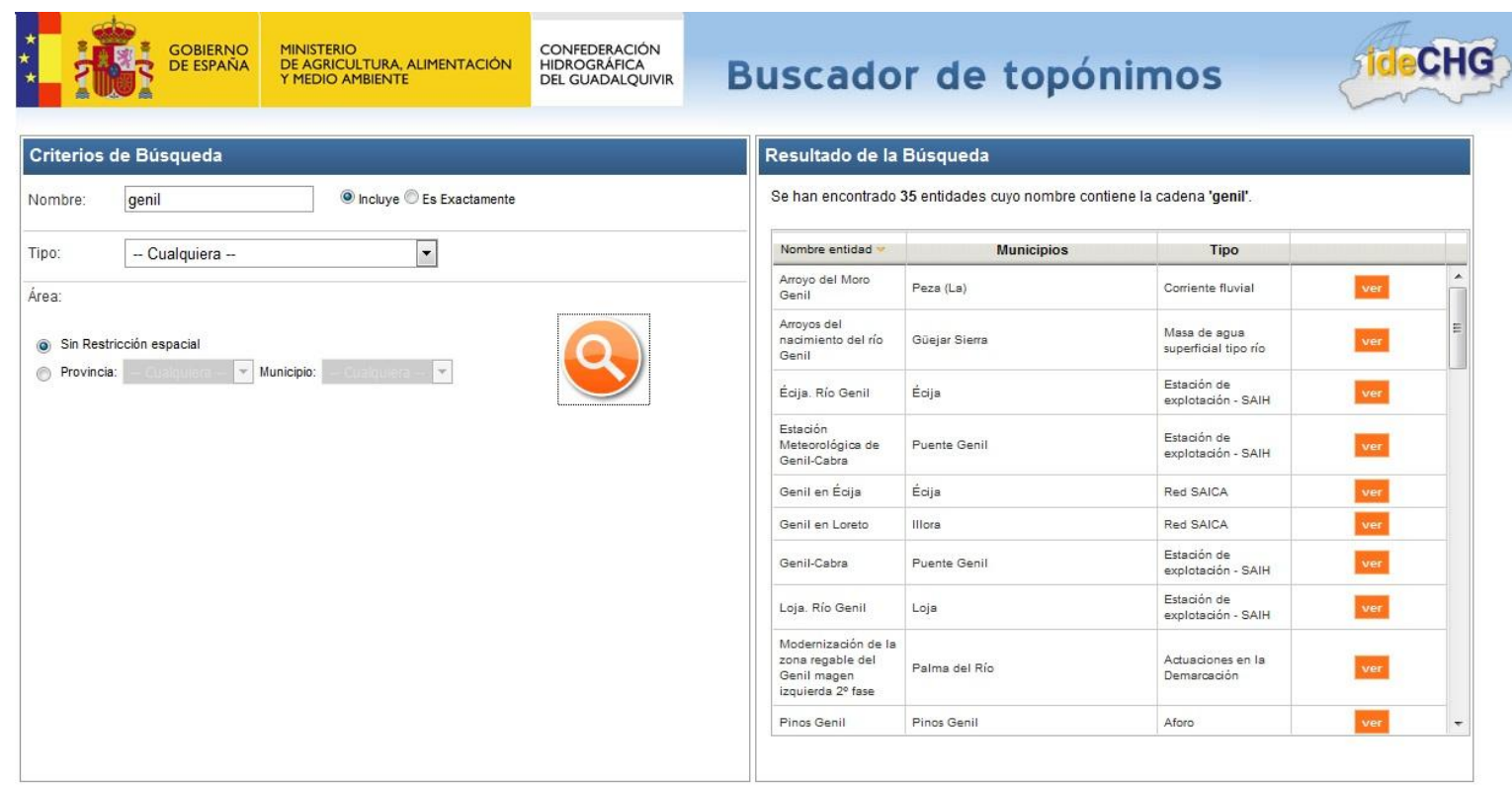

Figura 3. Buscador de topónimos de la IDE-CHG.

Fuente: elaboración propia.

Asimismo, el usuario tiene la opción de visualizar la entidad o topónimo de interés en el visualizador cartográfico. El método de representación sobre el mapa de las búsquedas se realiza atendiendo a las recomendaciones del Modelo de Nomenclátor de España (MNE), de tal forma que las corrientes fluviales, por ejemplo, se representan mediante un punto, el de la desembocadura; mientras que las entidades poligonales, como las unidades hidrogeológicas, se representan mediante el mínimo rectángulo envolvente.

El buscador de topónimos es una aplicación desarrollada en JavaEE con framework Spring, al igual que el visualizador cartográfico. Para realizar consultas espaciales hace uso de la aplicación 
González Rojas, D., Cifuentes Sánchez, V. J., Sancho Miró, A. (2019): "Las infraestructuras de datos espaciales al servicio de la gestión del agua. El caso de la Confederación Hidrográfica del Guadalquivir”, GeoFocus (Artículos), $n^{\circ}$ 24, p.3-17. ISSN: 1578-5157 http://dx.doi.org/10.21138/GF.548

Degree-wfs, que implementa el servicio WFS-G. Este estándar especifica cómo se deben implementar servicios de nomenclátor. Un servicio de nomenclator ofrece la posibilidad de localizar un fenómeno geográfico de un determinado nombre. WFS-G es un caso particular del servicio WFS en el que los criterios de localización de la información espacial vienen dados por búsquedas textuales (tesauros, topónimos, etc.).

\subsection{Servicios INSPIRE}

Los servicios OGC permiten localizar, visualizar y descargar la información geoespacial de la IDE-CHG, en cumplimiento de la Directiva 2007/2/CE. Pueden accederse desde cualquier lugar, necesitándose para ello software GIS específico, como ArcGIS, QGIS o gvSIG; o bien un visualizador web que permita conexiones a servicios externos. La Confederación Hidrográfica del Guadalquivir pone a disposición del ciudadano los siguientes geoservicios:

- Web Map Service (WMS). El servicio WMS permite, mediante el intercambio de peticiones y respuestas XML, publicar mapas a la carta a partir de datos georreferenciados, sin que se acceda a los datos en sí mismos, sólo a una representación gráfica de los mismos. Estos mapas pueden contener información de varias capas raster y/o vectoriales, superpuestas en un determinado orden modificable. La URL de acceso al servicio WMS es: http://idechg.chguadalquivir.es/inspire/wms.

- Web Feature Service (WFS). El servicio de entidades ofrece acceso de lectura a datos vectoriales utilizando GML como protocolo subyacente para realizar consultas espaciales, recuperar los datos y manipular la geometría. La URL de acceso al servicio WFS es: http://idechg.chguadalquivir.es/inspire/wfs.

- Web Coverage Service (WCS). El servicio de coberturas proporciona información raster con su semántica original. Es decir, permite el acceso no sólo a la imagen en sí (tal y como hace el servicio WMS), sino también a los valores o propiedades de la misma. La URL de acceso al servicio WCS es: http://idechg.chguadalquivir.es/inspire/wcs.

- Catalog Web Services (CSW). El servicio de localización permite conocer toda la información geoespacial que está disponible en este nodo IDE, así como acceder a sus metadatos. La URL de acceso al servicio CSW es: http://idechg.chguadalquivir.es/inspire/csw.

\section{Información espacial incluida en la IDE-CHG}

La IDE-CHG ofrece información geográfica de referencia de los ámbitos de las demarcaciones hidrográficas del Guadalquivir, Ceuta y Melilla. Esta información es producida en su mayoría por el organismo, aunque también se publican otros datos de interés cuya fuente de procedencia es externa, por lo que ha cobrado especial relevancia el establecimiento de políticas y acuerdos institucionales destinados a facilitar la disponibilidad, coordinación y acceso conjunto a información espacial procedente de diversas fuentes, lo que ha repercutido en una considerable disminución de costes de adquisición de datos y ha paliado, en cierta medida, los solapamientos de información y la duplicación de esfuerzos (Serrano, 2007). En este sentido los metadatos juegan un papel fundamental a la hora de identificar el origen y la calidad de la información. 
González Rojas, D., Cifuentes Sánchez, V. J., Sancho Miró, A. (2019): “Las infraestructuras de datos espaciales al servicio de la gestión del agua. El caso de la Confederación Hidrográfica del Guadalquivir”, GeoFocus (Artículos), $n^{\circ}$ 24, p.3-17. ISSN: 1578-5157 http://dx.doi.org/10.21138/GF.548

Paralelamente a las labores de desarrollo del sistema, la Confederación Hidrográfica del Guadalquivir ha efectuado las actualizaciones pertinentes sobre el modelo de datos, que han venido motivadas especialmente por la incorporación de nuevas funcionalidades y nuevas fuentes de información, tanto internas como externas. En este sentido, cabe destacar la recopilación periódica de series históricas de diferentes redes de control del organismo (piezómetros, manantiales, calidad de aguas superficiales, etc.) y la importancia de llevar a cabo una labor constante de definición de procedimientos de actualización y almacenamiento de toda la información geoespacial.

Toda la información recogida, almacenada y procesada es integrada en un modelo de datos común que permite relacionarla y darle coherencia. Dicho modelo controla el estado de cada fuente de información, su origen y las características que deben guiar su publicación. Antes de ser cargada en la geodatabase, la información recopilada es sometida a un riguroso proceso de calidad topológica, geométrica y temática. Igualmente, se generan los metadatos de las capas según las recomendaciones del GT-IDEE, así como el diccionario de datos que describe cada uno de los campos alfanuméricos o columnas de las entidades.

Dependiendo del formato en que se encuentra la información se realizan distintos controles. En los casos en los que se efectúa una labor de recopilación de información vectorial y ráster, se tienen en cuenta los siguientes aspectos:

- Viabilidad de la información. Se realiza un análisis previo de la aptitud de la información, seleccionando aquella que ha resultado de mayor interés.

- Según el formato de almacenamiento se presuponen una serie de errores posibles, como por ejemplo, errores topológicos en ficheros shapefile; aparición de polígonos sin cerrar y arcos discontinuos en ficheros CAD, etc.

- Aunque los datos hayan sido digitalizados en un determinado sistema de referencia y con una determinada proyección, se comprueba la existencia de ficheros de proyección o, en su defecto, de metadatos que contemplaran esta información.

- Generación de metadatos y diccionario de datos.

- Se examinan qué formatos son topológicos y cuáles no. Aquellos que lo son (por ejemplo, coberturas de ArcInfo) permiten obviar determinados errores en la cartografía.

En los casos de tablas en formato fichero o que forman parte de una base de datos, aparte de los atributos de determinadas entidades, dicha información puede contener geometría. En este caso, el procesado contempla:

- Generación de geometría a partir de datos alfanuméricos. Toda la cartografía se referencia al sistema geodésico definido por el European Terrestrial Reference System 1989 (ETRS89). Por su parte, la proyección cartográfica empleada es Universal Transverse Mercator (UTM) en su Huso 30.

- Vinculación con la información alfanumérica, contenida en tablas. Se comprueba que cada elemento gráfico disponga de un código identificador único, que sirva para establecer una relación entre la información gráfica y los atributos de los elementos.

- Generación de metadatos y diccionario de datos. 
González Rojas, D., Cifuentes Sánchez, V. J., Sancho Miró, A. (2019): “Las infraestructuras de datos espaciales al servicio de la gestión del agua. El caso de la Confederación Hidrográfica del Guadalquivir”, GeoFocus (Artículos), $n^{\circ}$ 24, p.3-17. ISSN: 1578-5157 http://dx.doi.org/10.21138/GF.548

Toda la información georreferenciada es debidamente tratada con el objeto de compatibilizarla con el Sistema de Información sobre el Agua para Europa (http://water.europa.eu/), en adelante WISE, de la Agencia Europea de Medio Ambiente. La información recopilada en el sistema WISE acerca del agua en España se realiza a través del Sistema Integrado de Información del Agua (www.mapama.gob.es/es/agua/temas/planificacion-hidrologica/sia-/), que ha sido diseñado para permitir y facilitar la comunicación electrónica de resultados a la Comisión Europea.

La IDE-CHG incluye información georreferenciada que permite describir las demarcaciones hidrográficas del Guadalquivir, Ceuta y Melilla, tanto desde el punto de vista del ámbito territorial como del físico y biótico, así como los marcos institucional y socioeconómico.

Para las aguas tanto superficiales como subterráneas se incluye información espacial sobre sus límites y localización, ecorregiones, tipos y condiciones de referencia. Además, se incorpora el inventario de los recursos superficiales y subterráneos incluyendo sus regímenes hidrológicos y las características básicas de calidad de las aguas (ver figura 4).

\section{Figura 4. Datos relacionados sobre las masas de agua.}

Fuente: elaboración propia.

A través de la IDE-CHG es posible consultar los usos y demandas existentes con una estimación de las presiones sobre el estado cuantitativo de las aguas, la contaminación de fuente puntual y difusa, incluyendo los usos del suelo y otras afecciones significativas de la actividad humana sobre los recursos hídricos. También se incluye la identificación y mapas de las zonas protegidas.

Finalmente, destacar la posibilidad de consultar las redes de control establecidas para el seguimiento del estado de las aguas. Mediante el uso de esta funcionalidad, se puede obtener información sobre las distintas estaciones pertenecientes a las diferentes redes de control y sus series temporales de medidas, obteniendo informes que incluyen gráficas.

En primer lugar, la herramienta le da la opción al usuario de elegir, por un lado, la red de control que le interese, y por otro, la estación concreta que desee consultar. Se puede observar cómo el mapa se refresca automáticamente, centrándose la vista en la estación seleccionada. 
González Rojas, D., Cifuentes Sánchez, V. J., Sancho Miró, A. (2019): “Las infraestructuras de datos espaciales al servicio de la gestión del agua. El caso de la Confederación Hidrográfica del Guadalquivir”, GeoFocus (Artículos), $n^{\circ}$ 24, p.3-17. ISSN: 1578-5157 http://dx.doi.org/10.21138/GF.548

A continuación, es posible consultar o bien la información de la estación o bien los históricos de datos. En el primer caso, la aplicación nos devuelve la información alfanumérica de la capa y los parámetros que mide la estación. En el segundo caso, existen varias posibilidades:

- Se pueden consultar los valores de un único parámetro, registrados en un rango de fechas. En este caso, es posible obtener un informe en pdf, con una gráfica y el listado de las medidas.

- También pueden compararse los valores de tres parámetros, registrados en un rango de fechas. En este caso, es igualmente posible obtener un informe en formato pdf.

- Finalmente, es posible obtener una lista de todos los valores medidos por la estación seleccionada, dentro de un rango de fechas que no sea superior a 12 años consecutivos.

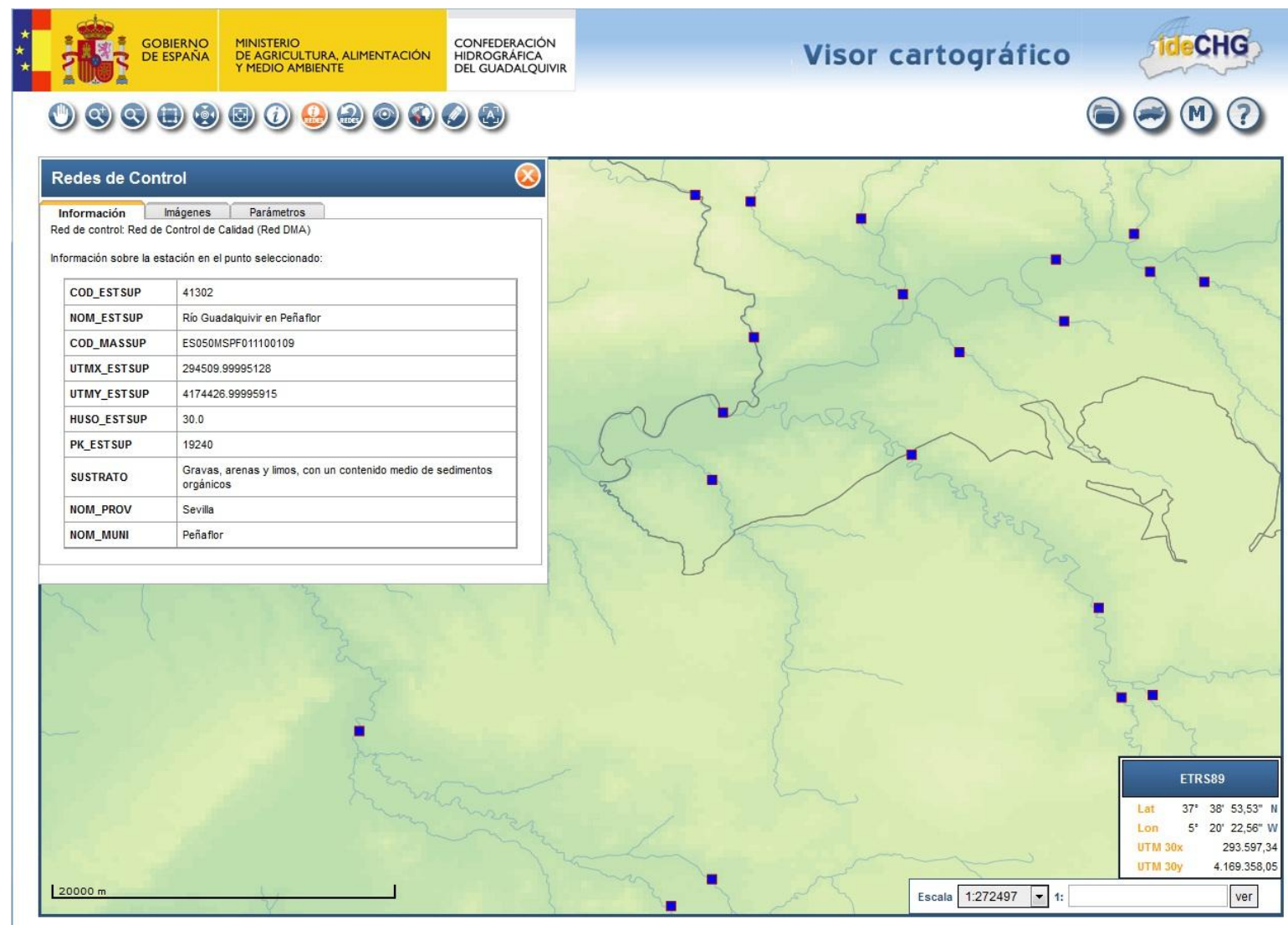

Figura 5. Consulta red de control de calidad de las aguas superficiales. Fuente: elaboración propia.

\section{Conclusiones}

Los trabajos para la creación del nodo IDE-CHG han sido dirigidos por la Oficina de Planificación Hidrológica de la Confederación Hidrográfica del Guadalquivir. Esta experiencia demuestra la utilidad de esta tecnología para el descubrimiento y difusión de la información, y la integración la misma con multitud de datos geográficos que faciliten su análisis. 
González Rojas, D., Cifuentes Sánchez, V. J., Sancho Miró, A. (2019): "Las infraestructuras de datos espaciales al servicio de la gestión del agua. El caso de la Confederación Hidrográfica del Guadalquivir”, GeoFocus (Artículos), $n^{\circ}$ 24, p.3-17. ISSN: 1578-5157 http://dx.doi.org/10.21138/GF.548

Los resultados obtenidos se han plasmado en la puesta en marcha de una infraestructura robusta orientada a:

- Generar una estructura que permita unificar y centralizar la información geográfica y alfanumérica básica de las demarcaciones del Guadalquivir, Ceuta y Melilla.

- Contribuir a reforzar la fiabilidad de los datos almacenados.

- Disponer de un nivel de información común para todos los usuarios.

- Simplificar y mejorar el acceso a la información a los usuarios de la organización y a toda la ciudadanía.

La ejecución de este plan ha permitido la puesta en marcha de un sistema flexible y escalable en cuanto a funcionalidades de consulta, análisis y gestión en un entorno Web, constituyéndose en la plataforma SIG corporativa del organismo. A través del geoportal, la Confederación Hidrográfica del Guadalquivir establece, gestiona y provee de acceso a una red de servicios que permite descubrir, localizar, visualizar y descargar los datos geográficos.

En definitiva, la IDE-CHG ofrece a los ciudadanos los servicios necesarios para visualizar y descubrir información geográfica producida por la Confederación Hidrográfica del Guadalquivir, a la vez que permite integrar los servicios publicados por otras instituciones. Estos servicios geográficos irán creciendo a medida que el organismo genere nueva información, que será ofrecida a través del Geoportal.

\section{Referencias bibliográficas}

Directiva 2007/2/CE del Parlamento Europeo y del Consejo de 14 de marzo de 2007 por la que se establece una infraestructura de información espacial en la Comunidad Europea (Inspire). Diario Oficial de la Unión Europea L 108, 25 de abril de 2007. [Consulta: 08-11-2016]. Disponible en http://eur-lex.europa.eu/legal-content/ES/ALL/?uri=OJ:L:2007:108:TOC.

Infraestructura de Datos Espaciales de Andalucía. [Consulta: 09-11-2016]. Disponible en www.ideandalucia.es.

Infraestructura de Datos Espaciales de España. [Consulta: 09-11-2016]. Disponible en www.idee.es

Infraestructura de Datos Espaciales de la Confederación Hidrográfica del Guadalquivir. [Consulta: 09-11-2016]. Disponible en www.chguadalquivir.es/idechg.

Internacional Organization for Standarization. [Consulta: 09-11-2016]. Disponible en www.iso.org.

Kropla, Bill (2005): Beginning MapServer Open Source GIS Development. Berkeley, California, USA, Editorial Apress, pp. 25.

Ley 14/2010, de 5 de julio, sobre las infraestructuras y los servicios de información geográfica en España [Consulta: 08-11-2016]. Disponible en https://www.boe.es/diario_boe/txt.php?id=BOE-A2010-10707.

MapServer. [Consulta: 10-11-2016]. Disponible en http://mapserver.org/es/.

Open Geospatial Consortium. [Consulta: 09-11-2016]. Disponible en www.opengeospatial.org. 


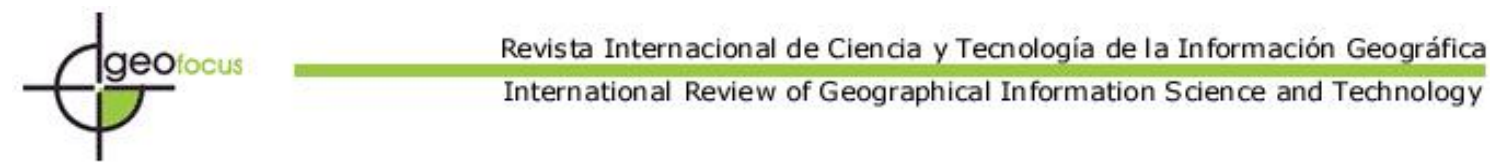

González Rojas, D., Cifuentes Sánchez, V. J., Sancho Miró, A. (2019): “Las infraestructuras de datos espaciales al servicio de la gestión del agua. El caso de la Confederación Hidrográfica del Guadalquivir”, GeoFocus (Artículos), $n^{\circ}$ 24, p.3-17. ISSN: 1578-5157 http://dx.doi.org/10.21138/GF.548

Open Source Geospatial Foundation. [Consulta: 09-11-2016]. Disponible en www.osgeo.org/.

Sistema de Información sobre el Agua para Europa. [Consulta: 09-11-2016]. Disponible en http://water.europa.eu.

Sistema Integrado de Información del Agua. [Consulta: 09-11-2016]. Disponible en www.mapama.gob.es/es/agua/temas/planificacion-hidrologica/sia-/.

Serrano Castillo, I. (2007): "Puesta en marcha del Sistema de Información Territorial de la Confederación Hidrográfica del Guadalquivir como nodo de la IDEE". IV Jornadas Técnicas de la IDE de España (JIDEE 07). [Consulta: 08-11-2016]. Disponible en Santiago de Compostela. http://www.idee.es/resources/presentaciones/JIDEE07/ARTICULOS JIDEE2007/articulo36.pdf

Zabala Ordóñez, A., Gómez Galán, P.J., Ramírez García, M.J. y Ripalda Marín, M.A. (2006): "Plan de Modernización de la Confederación Hidrográfica del Guadalquivir”. Tecnim@p, Sevilla. 
